\title{
Response of Acoustic Imaging Systems Using Convergent Leaky Waves to Cylindrical Flaws
}

\author{
NILGÜN GÜNALP AND ABDULLAH ATALAR, MEMBER IEEE
}

\begin{abstract}
Characterization of near-surface properties of materials by acoustic means is most conveniently done by surface-acoustic-waves (SAW) that are spatially confined to the surface. There are several techniques available to excite focused SAW's on the surfaces of nonpiezoelectric materials for imaging purposes. A conventional acoustic microscope lens excites focused SAW's on the object surface, but with a low efficiency. SAW can be excited with a high efficiency using conical wavefronts as obtained from a conical axicon. Such methods can be used in a scanning arrangement to generate images of surface inhomogeneities. In this paper, an analysis of imaging systems making use of convergent SAW's is given using angular spectrum approach and $T$ matrix formulation. Inhomogeneities on the object surface are assumed to be circular cylinders whose axes are perpendicular to the surface. The response of imaging systems to such defects is found as a function of defect position with respect to the focus point. Theoretical and experimental results are compared and are found to be in good agreement.
\end{abstract}

\section{INTRODUCTION}

$\mathrm{N}$ EAR SURFACE PROPERTIES of materials are important for their structural integrity. Nondestructive evaluation of these properties is usually carried out using acoustic techniques. Bulk waves fail to detect near surface faults due to the large reflection signal at the boundary. Surface acoustic waves (SAW) are frequently used for near surface evaluation of materials, because they are very sensitive to surface inhomogeneities like surface breaking cracks [1]. If the characterization is of primary concern rather than just detection of faults, an imaging system may be utilized. There are several imaging techniques available for this purpose. The scanning acoustic microscope (SAM) has been used successfully, because of its ability to excite surface waves [2]-[5]. The SAM excites other wave modes like generalized Lamb waves, which may be more sensitive for certain kind of defects [6]. The disadvantage of SAM is that all such modes are excited simultaneously and each with a low efficiency. The efficiency can be increased by use of other configurations. Smith $e t$ al. [7] proposed to use a semicircular disk transducer with

Manuscript received July 1, 1988; revised November 21, 1988: accepted December 5, 1988.

N. Günalp is with the Electrical and Electronics Engineering Department, Middle East Technical University, Ankara, Turkey 06531.

A. Atalar is with the Electrical and Electronics Engineering Department, Bilkent University, Ankara, Turkey 06572

IEEE Log Number 8927861. dition to excite focused SAW's. Nongaillard et al. [8] used a tilted cylindrical lens to excite focused SAW. Jen et al. [9], Cielo et al. [10], [11] and Maldague et al. [12] used a focused optical ring to generate SAW on the test sample. Köymen and Atalar introduced a method of focusing SAW's on the surface of nonpiezoelectric materials by using a new type of conical axicon [13]. Ayter [14] achieved the same result, using a conical transducer. Robbins et al. [15] proposed to use the scanning laser acoustic microscope with SAW's for thin-film characterization. Such systems allow one to scan the surface by a diffraction limited spot and thus to image the surface properties of the materials.

The purpose of this paper is to present a theoretical study of the imaging systems utilizing focused leaky SAW, and their response to certain kind of defects. In particular, circular cylindrical inhomogeneities axes perpendicular to the surface are considered. Scattering of acoustic plane waves from cylinders has been investigated extensively [16], [17], but, there are very few studies on scattering of a bounded acoustic beam by a cylinder [18]. In this paper the scattering of the SAW from this cylinder is formulated with some approximations. The surface wave incident upon the inhomogeneity is initially found as an angular spectrum of plane waves. But, for the application of the boundary conditions at the cylindrical surface, the incident field has to be transformed into a form of superposition of cylindrical waves. Similarly, the scattered field, which is found in the form of outgoing cylindrical SAW's, is converted back to a plane wave spectrum in order to complete the formulation. These transformations between the angular spectrum of plane waves and the cylindrical wave expansions constitute the crucial point of the formulation. At the end, a formula is obtained for the transducer output voltage in terms of the position and the radius of the cylinder, and it is suitable for computer evaluation. By considering various locations for the cylinder, the sensitivity of the system around the focal point is studied. Also, by comparing the output voltages for cylinders of different radii, sensitivity of the system to the size of the inhomogeneity is examined. The numerical results are found to be in agreement with the experimental observations. 


\section{Description of the Imaging System}

As a representative of SAW imaging systems, we consider the conical axicon focusing system [13]. This configuration is simple to fabricate, and a high bulk-to-SAW conversion efficiency can be obtained. Here, we give a brief review of the principle of operation of this imaging system. As shown in Fig. 1, an acoustic beam generated by the piston transducer is obliquely incident on the concave parabolic cylindrical surface of the mirror. If the incidence angle is high enough, all of the incident power will be reflected, and no bulk wave will be excited in the solid mirror block. The wavefronts of the reflected wave are conical [19], and the axis of the cones coincides with the focal line of the parabolic cylinder that is perpendicular to the object surface under test. The intersection of the conical wavefronts with the surface of the material is always circular. Since the mirror has finite aperture, the reflected wavefront will be a section of the conical surface, and hence the intersection with the material surface will be a circular arc rather than a complete circle.

The incidence angle of the beam created by the transducer is adjusted such that the reflected wave is incident on the material surface at the Rayleigh critical angle. By this process all the energy in a conical wavefront is converted to a single circularly converging wavefront of the surface wave. This surface wave will start to leak into the liquid medium as a bulk wave, but almost none of the incident power will return to the transducer if the material surface is flawless. However, when there is a surface wave reflector at the focal spot, the transducer will receive an appreciable power: the scattered surface waves will be circularly divergent and they will leak into the liquid medium to reconstruct the conical wavefronts that originally created the surface wave. This backward conical wavefront will get collimated in the transducer direction after being reflected from the parabolic mirror. If the inhomogeneity is not exactly at the surface wave focus, the transducer output voltage will be reduced.

\section{Formulation}

Consider the geometry shown in Fig. 2. The $(r, s, t)$ coordinate system will be used to represent the bulk waves transmitted and received by the transducer, and $(x, y, z)$ coordinates will be used for the surface waves in the solid. The origin of the $(x, y, z)$ coordinate system is chosen at the focal point. Inside the solid a circular cylindrical inhomogeneity is considered. This cylinder extends up to the interface and it is centered at $\left(x_{0}, y_{0}\right)$ with $y_{0}>0$ as demonstrated in Fig. 1.

The formulation presented below employs two-dimensional potentials $u^{+}(x, y)$ and $u^{-}(x, y)$ to represent the incident and scattered surface waves. It is shown in the Appendix that such two-dimensional potentials (either in the form of angular spectrum of plane waves or in the form of cylindrical wave expansions) are sufficient to represent completely the three-dimensional characteristics of SAW's by suitably multiplying the depth variation ( $z$-de-

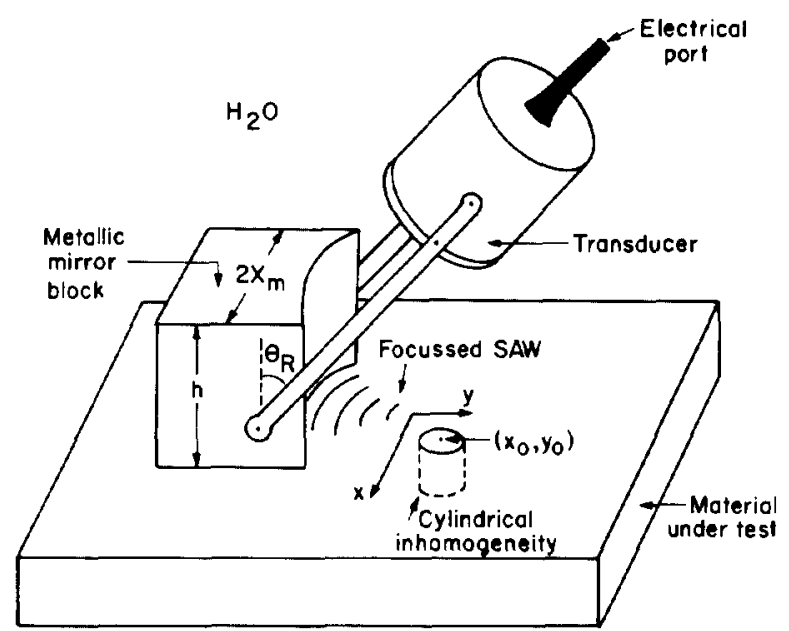

Fig. 1. Conical axicon geometry to obtain convergent SAW.

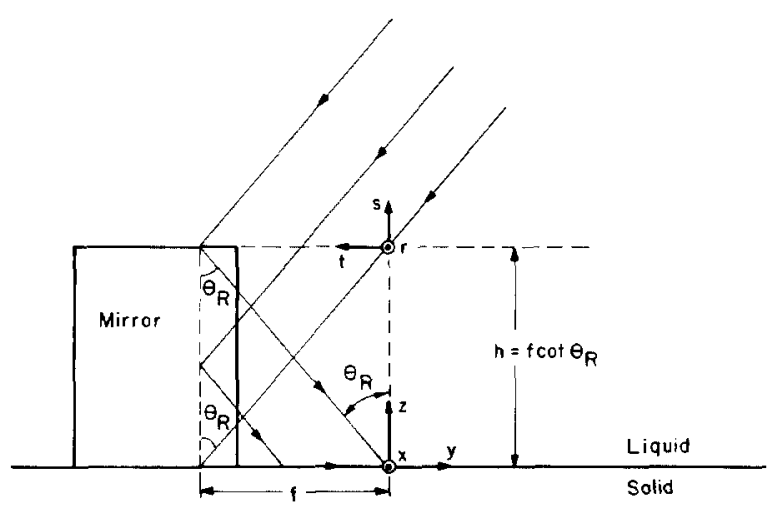

Fig. 2. Description of coordinates used in formulation.

pendence $)$. A time dependence of $\exp (-j \omega t)$ is assumed in the formulation.

\section{Surface Wave Field at the Focus}

Let the velocity potential associated with the incident bulk wave be represented by $u_{b}^{+}(r, s)$ at the $r-s$ plane. Since the parabolic mirror acts like a Fourier transform operator in one direction [20], and the bulk to surface wave conversion phenomenon acts as an integrator in the other dimension [21], the surface wave field along $x$-axis and propagating in the $+y$ direction, $u_{f}^{+}(x)$, can be written as [22]

$$
\begin{aligned}
u_{f}^{+}(x)= & \int_{-\infty}^{\infty}\left\{\int_{-\infty}^{+\infty} u_{b}^{+}(r, s) P(r, s) d s\right\} \\
& \cdot \exp \left[-j\left(k_{R} / f\right) r x\right] d r
\end{aligned}
$$

where $k_{R}=2 \pi / \lambda_{R}$ is the real part of the leaky Rayleigh wave number, $f$ is the focal length, and $P(r, s)$ is the pupil function due to finite size of the parabolic mirror. $P(r, s)$ is obtained from a projection of the mirror surface onto the $r-s$ plane: a rectangle of width $2 x_{m}$ and height $f \cot \theta_{R}$, where $\theta_{R}$ is the Rayleigh angle. The pupil function given below also includes the phase and attenuation 
factors that arise as the wave travels from the mirror surface up to the focal point. We have

$$
\begin{aligned}
& P(r, s) \\
& =\left\{\begin{array}{l}
\exp \left[-\alpha_{T}\left(f-s \tan \theta_{R}\right)\right] \exp \left(j k_{R} s \cot \theta_{R}\right), \\
\text { for }-x_{m}<r<x_{m}, 0<s<f \cot \theta_{R} \\
0, \quad \text { otherwise, }
\end{array}\right.
\end{aligned}
$$

where $\alpha_{T}=\alpha_{D}+\alpha_{L} ; \alpha_{D}$ and $\alpha_{L}$ are the dissipation and leak rates, respectively, of the surface wave.

\section{Field Incident Upon the Cylinder}

By using the angular spectrum approach [23], the field in the region $y>0$ can be completely determined by the field distribution at $y=0$, i.e., along $x$-axis. For this purpose, we first define the angular spectrum, $U_{f}^{+}\left(k_{x}\right)$, of the surface wave field, $u_{f}^{+}(x)$, by

$$
U_{f}^{+}\left(k_{x}\right)=\mathcal{F}\left\{u_{f}^{+}(x)\right\}=\int_{-\infty}^{+\infty} u_{f}^{+}(x) \exp \left(-j k_{x} x\right) d x
$$

where $\mathcal{F}$ is the Fourier transform operator. Then the angular spectrum at a position $y>0$ can be found from

$$
U^{+}\left(k_{x}, y\right)=U_{f}^{+}\left(k_{x}\right) \exp \left(j k_{y} y\right)
$$

This equation describes the propagation of the angular spectrum in the $+y$ direction. Since $u^{+}(x, y)$ and $U^{+}\left(k_{x}\right.$, $y$ ) form a Fourier transform pair, the field $u^{+}(x, y)$ for $y$ $>0$ can then be expressed as

$$
\begin{gathered}
u^{+}(x, y)=1 /(2 \pi) \int_{-\infty}^{+\infty} U^{+}\left(k_{x}, y\right) \exp \left(j k_{x} x\right) d k_{x}, \\
y \geq 0
\end{gathered}
$$

or

$$
\begin{aligned}
u^{+}(x, y)= & 1 /(2 \pi) \int_{-\infty}^{+\infty} U^{+}\left(k_{x}\right) \\
& \cdot \exp \left[j\left(k_{x} x+k_{y} y\right)\right] d k_{x}, \quad y \geq 0
\end{aligned}
$$

where $k_{x}$ and $k_{y}$ are related by $k_{x}^{2}+k_{y}^{2}=k^{2}$, and $k$ is the complex wave number, $k=k_{R}+j \alpha_{T}$. We can express $k_{y}$ as $k_{y}=\left(k^{2}-k_{x}^{2}\right)^{1 / 2}$ with $\operatorname{Im}\left\{k_{y}\right\}>0$.

One can directly combine (1) and (3), and obtain the following expression for $U_{f}^{+}\left(k_{x}\right)$ in terms of an arbitrary incident field $\boldsymbol{u}_{b}^{+}(r, s)$ as

$$
U_{f}^{+}\left(k_{x}\right)=\left.f \lambda_{R} \int_{-\infty}^{+\infty}\left[u_{b}^{+}(r, s) P(r, s)\right]\right|_{r=-f k_{x} / k_{R}} d s .
$$

\section{Field Scattered by the Cylinder}

To formulate the scattering, we first introduce a primed coordinate system $\left(x^{\prime}, y^{\prime}\right)$ with the origin at the center of the cylinder, i.e.,

$$
x^{\prime}=x-x_{0}, \quad y^{\prime}=y-y_{0}
$$

as shown in Fig. 3. To simplify the notation, we also introduce the vectors $r=(x, y), r^{\prime}=\left(x^{\prime}, y^{\prime}\right), r_{0}=\left(x_{0}\right.$, $\left.y_{0}\right), \boldsymbol{k}=\left(k_{x}, k_{y}\right)$. Then the field incident upon the cylinder can be written as

$$
\begin{gathered}
u^{+}\left(\boldsymbol{r}^{\prime}\right)=1 /(2 \pi) \int_{-\infty}^{+\infty} U_{f}^{+}\left(k_{x}\right) \exp \left(j k \bullet r_{0}\right) \\
\cdot \exp \left(j k \bullet \boldsymbol{r}^{\prime}\right) d k_{x}
\end{gathered}
$$

or

$$
\begin{gathered}
u^{+}\left(\boldsymbol{r}^{\prime}\right)=1 /(2 \pi) \int_{-\infty}^{+\infty} A\left(k_{x}\right) \exp \left(j k \bullet \boldsymbol{r}^{\prime}\right) d k_{x}, \\
y^{\prime} \geq-y_{0}
\end{gathered}
$$

where

$$
A\left(k_{x}\right)=U_{f}^{+}\left(k_{x}\right) \exp \left(j k \bullet r_{0}\right) .
$$

Let $u^{-}\left(\boldsymbol{r}^{\prime}\right)$ denote the field scattered by the cylinder. Then, the total field outside the cylinder must be given by

$$
u\left(\boldsymbol{r}^{\prime}\right)=u^{-}\left(\boldsymbol{r}^{\prime}\right)+u^{-}\left(\boldsymbol{r}^{\prime}\right) .
$$

In order to relate the incident and the reflected fields by the boundary conditions on a cylindrical surface $\rho^{\prime}=a$, it is necessary to express these fields in terms of cylindrical wave functions. The incident field that will be evaluated at $\rho^{\prime}=a$ can be expressed in terms of regular cylindrical wave functions as

$$
u^{+}\left(\boldsymbol{r}^{\prime}\right)=\sum_{n=-\infty}^{+\infty} K_{n} \exp \left(j n \phi^{\prime}\right) J_{n}\left(k \rho^{\prime}\right)
$$

and the scattered field can be expanded in terms of outgoing cylindrical wave functions as

$$
\begin{gathered}
u^{-}\left(\boldsymbol{r}^{\prime}\right)=\sum_{n=-\infty}^{+\infty} B_{n} \exp \left(j n \phi^{\prime}\right) H_{n}^{(1)}\left(k \rho^{\prime}\right), \\
\rho^{\prime} \geq a .
\end{gathered}
$$

The relationship between $\left\{K_{n}\right\}$ of (9) and $A\left(k_{x}\right)$ of (7) can be established by substituting the following transformation formula [24] into (7):

$$
\begin{aligned}
\exp \left(j k \bullet r^{\prime}\right)= & \sum_{n=-\infty}^{+\infty} \exp (j n \pi / 2) \\
& \cdot \exp \left(j n \phi^{\prime}\right) J_{n}\left(k \rho^{\prime}\right)\left(\frac{k_{x}-j k_{y}}{k}\right)^{n} .
\end{aligned}
$$

This yields

$$
\begin{aligned}
K_{n}= & 1 /(2 \pi) \exp (j n \pi / 2) \\
& \cdot \int_{-\infty}^{+\infty} A\left(k_{x}\right)\left(\frac{k_{x}-j k_{y}}{k}\right)^{n} d k_{x} .
\end{aligned}
$$




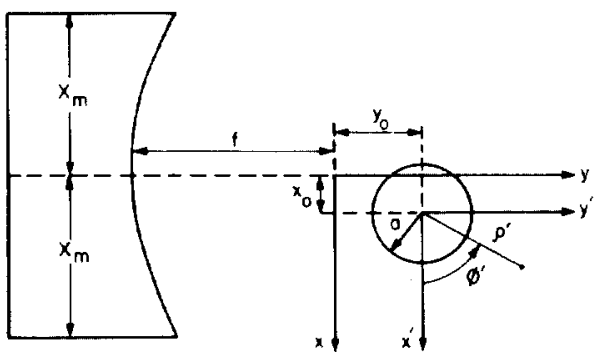

Fig. 3. Top view depicting primed coordinate system.

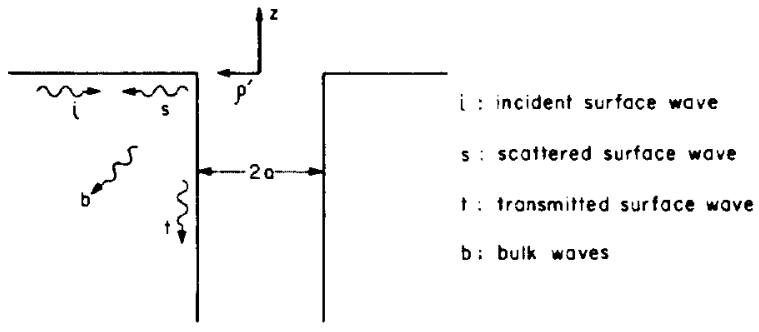

Fig. 4. Various wave components in SAW scattering problem.

The next step in the formulation is to express $\left\{B_{n}\right\}$, the expansion coefficients of the scattered wave, in terms of $\left\{K_{n}\right\}$. It is well-known that [25] when a surface wave is incident upon a scatterer, in general, a scattered surface wave, a transmitted surface wave and reflected bulk waves will be excited as demonstrated in Fig. 4.

We would like to consider a cylindrical cavity as the inhomogeneity, because the experimental results are obtained easily with cavities. Although only the scattered surface wave, $(s)$, will induce an output voltage at the transducer, for an exact solution of the problem all possible field components should be taken into account. Such an exact formulation is quite complicated [26]-[31], therefore a simple approximate method will be applied: Since the finite value of $c_{44}$ makes the surface of the cylinder a relatively hard boundary in the radial direction, the total surface wave along the interface $(i+s)$ will be forced to satisfy the rigid-boundary condition $\left(v_{\rho^{\prime}}=0\right.$ at $\rho^{\prime}=a$ ). Use of the free-boundary condition is not appropriate here, it approximates a cavity in a liquid medium where $c_{44}$ is zero. Even though the inhomogeneity is a cavity, it is better approximated in the scalar approximation by a rigid cylinder. In other words the reflection problem we are considering is the rigid cylinder case. But we will use the theoretical results to compare with experimental observations obtained with cavities. This is a good approximation for small size cylinders. This approximate formulation does not give the actual "ratio" of the scattered surface wave to the incident (for an infinite edge reflector it estimates a total reflection). However, it can quite satisfactorily be used to compare the scattered fields (or transducer output voltages) due to various cylinders of different locations and sizes. When the boundary condition

$$
\left.\frac{\partial}{\partial \rho^{\prime}}\left\{u^{+}\left(\boldsymbol{r}^{\prime}\right)+u^{-}\left(\boldsymbol{r}^{\prime}\right)\right\}\right|_{\rho^{\prime}=a}=0
$$

is applied with $u^{+}\left(\boldsymbol{r}^{\prime}\right)$ and $u^{-}\left(\boldsymbol{r}^{\prime}\right)$ as given by (9) and (10), respectively, one obtains

$$
B_{n}=T_{n} K_{n}
$$

where

$$
T_{n}=-\dot{J}_{n}(k a) / \dot{H}_{n}^{(1)}(k a) .
$$

Note that this procedure is a standard T-matrix formulation [32].

The scattered surface wave, which was expressed in cylindrical form in (10), must now be written as

$$
\begin{aligned}
u^{-}(x, y)= & 1 /(2 \pi) \int_{-\infty}^{+\infty} U_{f}^{-}\left(k_{x}\right) \\
& \cdot \exp \left[j\left(k_{x} x-j k_{y} y\right)\right] d k_{x}
\end{aligned}
$$

for points $y \leq y_{0}$. Note that $U_{f}^{-}\left(k_{x}\right)$ corresponds to the returning angular spectrum at $y=0$. To obtain such a representation, the following transformation formula [33] will be used:

$$
\begin{aligned}
& \exp \left(j n \phi^{\prime}\right) H_{n}^{(1)}\left(k \rho^{\prime}\right) \\
&= \frac{\exp (-j n \pi / 2)}{\pi} \int_{-\infty}^{+\infty}\left(\frac{k_{x}-j k_{y}}{k}\right)^{n} \\
& \cdot \exp \left[j\left(k_{x} x^{\prime}-k_{y} y^{\prime}\right)\right] / k_{y} d k_{x}
\end{aligned}
$$

for $y^{\prime} \leq 0$. Substitution of (15) into (10), and use of (6) yield

$$
\begin{aligned}
u^{-}(x, y)= & \int_{-\infty}^{+\infty} \sum_{n=-\infty}^{+\infty} B_{n} \frac{\exp (-j n \pi / 2)}{\pi k_{y}}\left(\frac{k_{x}-j k_{y}}{k}\right)^{n} \\
& \cdot \exp \left[-j\left(k_{x} x_{0}-k_{y} y_{0}\right)\right] \\
& \cdot \exp \left[j\left(k_{x} x-k_{y} y\right)\right] d k_{x}
\end{aligned}
$$

for $y \leq y_{0}$. Thus, $U_{f}^{-}\left(k_{x}\right)$ of (14) is recognized to be

$$
\begin{aligned}
U_{f}^{-}\left(k_{x}\right)= & \sum_{n=-\infty}^{+\infty} 2 B_{n} \frac{\exp (-j n \pi / 2)}{k_{y}}\left(\frac{k_{x}-j k_{y}}{k}\right)^{n} \\
& \cdot \exp \left[-j\left(k_{x} x_{0}-k_{y} y_{0}\right)\right] .
\end{aligned}
$$

\section{Transducer Output Voltage}

The scattered surface wave $u^{-}(x, y)$ travels in the backward direction and leaks into the liquid. This leak field gets collimated in the transducer direction after being reflected from the mirror. The field incident upon the transducer, $u_{b}^{-}(r, s)$, can be obtained by [22]

$$
u_{b}^{-}(r, s)=\alpha_{L} P(r, s) \int_{-\infty}^{+\infty} u_{f}^{-}(x) \exp \left(-j \frac{k_{R}}{f} r x\right) d x
$$

where $u_{f}^{-}(x)=u^{-}(x, 0)$ is the scattered surface wave field along the $x$-axis. Equation (17) "transforms" $u_{f}^{-}(x)$ to $u_{b}^{-}(r, s)$ correctly, since $P(r, s)$ includes the phase shift and the attenuation experienced by the wave as it travels from the focal spot to the mirror surface; it also 
limits the beam in both directions and the integral in (17) represents the Fourier transforming action of the cylindrical mirror. When combined with $U_{f}^{-}\left(k_{x}\right)=\mathscr{F}\left\{u_{f}^{-}(x)\right\}$ as in (3), (17) yields

$$
u_{b}^{-}(r, s)=\alpha_{L} P(r, s) U_{f}^{-}\left(\frac{k_{R}}{f} r\right) .
$$

Finally, since $u_{b}^{-}(r, s)$ is to be received by the same transducer as generated the incident field, $u_{b}^{+}(r, s)$, the output voltage of the transducer can be expressed [34] approximately as

$$
V=\int_{-\infty}^{+\infty} \int_{-\infty}^{+\infty} u_{b}^{+}(r, s) u_{b}^{-}(r, s) d r d s
$$

By combining (19), (18), (16), (12), (11), (8), (5) and (2), the transducer output voltage can be expressed, after some manipulations, in the following compact form:

$$
\begin{aligned}
V= & \frac{2 \alpha_{L} f^{2} \exp \left(-2 \alpha_{T} f\right)}{k_{R}^{2}} \sum_{n=-\infty}^{+\infty} T_{n} P_{n}\left(r_{0}\right) Q_{n}\left(r_{0}\right) \\
P_{n}\left(r_{0}\right)= & \int_{-\left(x_{m} / f\right) k_{R}}^{\left(x_{m} / f\right) k_{R}} g\left(k_{x}\right) \exp \left[j\left(k_{x} x_{0}+k_{y} y_{0}\right)\right] \\
& \cdot\left(\frac{k_{x}-j k_{y}}{k}\right)^{n} d k_{x} \\
Q_{n}\left(r_{0}\right)= & \int_{-\left(x_{m} / f\right) k_{R}}^{\left(x_{m} / f\right) k_{R}} g\left(k_{x}\right) \exp \left[-j\left(k_{x} x_{0}-k_{y} y_{0}\right)\right] \\
& \cdot\left(\frac{k_{x}-j k_{y}}{k}\right)^{n} d k_{x} / k_{y}
\end{aligned}
$$

where

$$
\begin{aligned}
g\left(k_{x}\right)= & \int_{0}^{f \cot \theta_{R}} u_{b}^{+}\left(f k_{x} / k_{R}, s\right) \exp \left(\alpha_{T} s \tan \theta_{R}\right) \\
& \cdot \exp \left(j k_{R} s \cot \theta_{R}\right) d s
\end{aligned}
$$

and $k_{y}=\left(k^{2}-k_{x}^{2}\right)^{1 / 2}, k=k_{R}+j \alpha_{T}$, and $T_{n}$ is as given by (13). Note that, (20), (21) and (22) can be used to calculate the response of the system under any excitation by the transducer, by merely substituting $u_{b}^{+}(r, s)$ into the $g\left(k_{x}\right)$ expression given by (23).

\section{A Special Case}

As the incident bulk wave transmitted by the transducer, assume a unity amplitude uniform plane wave with no $r$-dependence. In this case, the field distribution at the $r-s$ plane can be expressed as

$$
u_{b}^{+}(r, s)=\exp \left[-j k_{R} s \cot \theta_{R}\right]
$$

since there is an angle between the $r-s$ plane and the plane wavefront. One can directly substitute (24) into (23) and evaluate $g\left(k_{x}\right)$ as

$$
g\left(k_{x}\right)=\left[\exp \left(\alpha_{T} f\right)-1\right] / \alpha_{T} \tan \theta_{R}
$$

which is a constant. Thus the expression for the output voltage is considerably simplified. Several observations can be made by considering some of the intermediate steps of the formulation for this special case of plane wave incidence. For example, when $u_{b}^{+}(r, s)$ is multiplied by $P(r, s)$, the linear phase factors cancel, indicating that all the rays reach the focal point in phase. Substitution of (2) and (24) into (1) result in the following focal field distribution:

$$
\begin{aligned}
u_{f}^{+}(x)= & \frac{2\left[1-\exp \left(-\alpha_{T} f\right)\right] x_{m} \cot \theta_{R}}{\alpha_{T}} \\
& \cdot \frac{\sin \left[\left(x_{m} / f\right) k_{R} x\right]}{\left(x_{m} / f\right) k_{R} x} .
\end{aligned}
$$

This expression shows that the lateral dependence of the focal field is a "sinc" function.

The corresponding angular spectrum of surface waves at $y=0$ is then found as

$U_{f}^{+}\left(k_{x}\right)=\left\{\begin{array}{c}f \lambda_{R}\left[1-\exp \left(-\alpha_{T} f\right)\right] /\left(\alpha_{T} \tan \theta_{R}\right), \\ \left|k_{x}\right| \leq x_{m} k_{R} / f \\ 0, \quad \text { otherwise. }\end{array}\right.$

Note that the angular spectrum at the focal line has a constant value.

The transducer output voltage under plane wave insonification can be written, by introducing a suitable normalization voltage, in the following form:

$$
V=\frac{V_{\text {edge }}}{2 \pi k_{R}\left(x_{m} / f\right)} \sum_{n=-\infty}^{+\infty} T_{n} P_{n}\left(r_{0}\right) Q_{n}\left(r_{0}\right)
$$

where

$$
\begin{aligned}
P_{n}\left(r_{0}\right)= & \int_{-\left(x_{m} / f\right) k_{R}}^{\left(x_{m} / f\right) k_{R}} \exp \left[j\left(k_{x} x_{0}+k_{y} y_{0}\right)\right] \\
& \cdot\left(\frac{k_{x}-j k_{y}}{k}\right)^{n} d k_{x} \\
Q_{n}\left(\boldsymbol{r}_{\mathbf{0}}\right)= & \int_{-\left(x_{m} / f\right) k_{R}}^{\left(x_{m} / f\right) k_{R}} \exp \left[-j\left(k_{x} x_{0}-k_{y} y_{0}\right)\right] \\
& \cdot\left(\frac{k_{x}-j k_{y}}{k}\right)^{n} d k_{x} / k_{y} .
\end{aligned}
$$

$V_{\text {edge }}$ corresponds to the transducer output voltage when there is an infinitely long edge (plane reflector) along the $x$-axis. $V_{\text {edge }}$ is calculated below and it can be used as a normalization factor, i.e., numerical calculations can be done for $V / V_{\text {edge }}$.

To find the output voltage corresponding to a perfect SAW reflector we assume that the incident and scattered fields given by (4) and (14) satisfy the same type of rigid boundary condition

$$
\left.\frac{\partial}{\partial y}\left\{u^{+}(x, y)+u^{-}(x, y)\right\}\right|_{y=0}=0 .
$$


This directly gives $U_{f}^{-}\left(k_{x}\right)=U_{f}^{+}\left(k_{x}\right)$, which means a total reflection. In this case one finds

$$
V_{\text {edge }}=2 x_{m} \alpha_{L} \lambda_{R} f\left(\frac{1-\exp \left(-\alpha_{T} f\right)}{\alpha_{T} \tan \theta_{R}}\right)^{2} .
$$

This expression shows the factors affecting the output voltage with a perfect SAW reflector at the focal line.

\section{Numerical Results}

For numerical calculations, we will assume a uniform plane wave incidence. For this case, (27) gives the transducer output voltage in terms of the center $\boldsymbol{r}_{0}=\left(x_{0}, y_{0}\right)$ and the radius, $a$, of the scattering cylinder. The integrals $P_{n}$ and $Q_{n}$ given by (28) and (29) depend only on the position of the cylinder, whereas the coefficient $T_{n}$ depends only on the radius. This situation is quite advantageous during the numerical calculations. Because, these integrals, once evaluated at a certain $\left(x_{0}, y_{0}\right)$ position, can be stored and be used subsequently for cylinders of different radii centered at the same point. We put the integrals $P_{n}$ and $Q_{n}$ into a form more suitable for numerical integration by using a simple change of variables:

$$
p=k_{x} /\left[\left(x_{m} / f\right) k_{R}\right] .
$$

When this is done it is observed that for the calculation of $V / V_{\text {edge }}$, the only parameters that must be specified are

$$
x_{m} / f, \alpha_{T} / k_{R}, k_{R} x_{0}, k_{R} y_{0} \text { and } k_{R} a \text {. }
$$

The integrals are evaluated using Simpson's 1/3 Rule, and they converge quite easily. The necessary number of integration points increases as $n$ and/or the distance of the center from focal point increases. For the calculation of $T_{n}$, the derivatives of the Bessel and Hankel functions of the complex argument

$$
k a=k_{R} a\left(1+j \alpha_{T} / k_{R}\right)
$$

are generated. For small cylinders with $k_{R} a<1$, the terms with $|n| \leq 3$ in the summation of (27) are sufficient. As the radius increases, more and more terms should be included. The computer program guarantees the convergence of the integrals and the summation automatically.

In order to compare our results with the previously published experimental results [19], we chose $x_{m} / f=0.74$ in all of the calculations given below. The ratio $\alpha_{T} / k_{R}$ is equal to 0.03 for aluminum, and this value is used in all of the presented results, except in Fig. 5, where several $\alpha_{T} / k_{R}$ ratios are considered.

The first set of results given in Fig. 5 corresponds to a small cylinder $\left(k_{R} a=0.5\right.$, diameter $\left.\simeq 0.16 \lambda_{R}\right)$ moved along the $y$-axis to see the axial sensitivity of the imaging system. Here the results are normalized by the transducer output voltage, $V_{f}$, obtained when the cylinder is centered at the focus. Curves corresponding to different $\alpha_{T} / k_{R}$ ra-

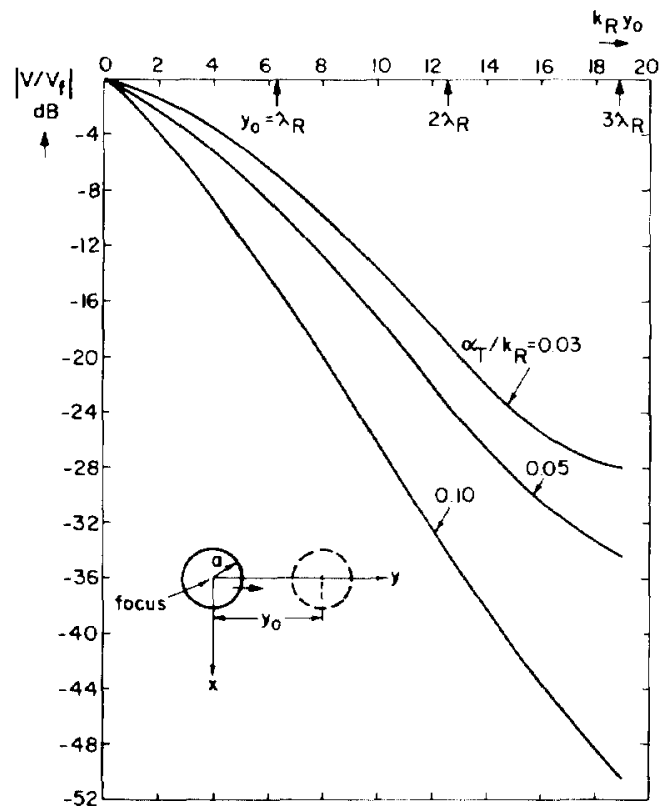

Fig. 5. Magnitude of normalized transducer output voltage as function of axial position of cylinder for several $\alpha_{T} / k_{R}$ values. $x_{m} / f=0.74, k_{R} a=$ $0.5, x_{0}=0.0, V_{f}=$ output voltage when cylinder is centered at focus. $V_{f}$ is $12.73 \mathrm{~dB}$ below $V_{\text {edge }}$

tios are presented. In each case it is seen that as the scatterer moves away from the focal point, the output voltage decreases monotonically. For the case of $\alpha_{T} / k_{R}=0.03$ (A1), 20-dB drop at the output voltage occurs at $k_{R} y_{0}=$ 13 that corresponds to a distance $y_{0} \simeq 2 \lambda_{R}$ from the focal point. This result is in close agreement with our experimental observations. It is interesting to note that as the leak rate is increased the response of the system in the $y$ direction gets sharper, i.e., the resolution in the $y$ direction increases.

To examine the sensitivity of the system along the $x$ direction (lateral direction), several calculations are carried out. First, a very small cylinder $\left(a=\lambda_{R} / 20\right)$ is moved along the $x$-axis (center at the $x$-axis) as shown in Fig. 6, and the normalized output voltage (in $\mathrm{dB}$ ) is plotted as a function of the separation of the cylinder center from the focal point. It is observed that $\left|V / V_{f}\right|$ versus $x_{0}$ curve closely follows the variations of the incident field distribution along $x$-axis $\left(u_{f}^{+}(x)\right.$, as given by (25)). $\left|u_{f}^{+}(x)\right|$ is also plotted in Fig. 6 after being normalized by its value at $x=0$. It is seen that $\left|V / V_{f}\right|$ is nearly equal to the square of the $\left|u_{f}^{+}\right|$(double the $\mathrm{dB}$ values) except for the peaks marked with $(*)$. Those peaks occur in the figure near the zero crossings of the sinc function. These unexpected peaks do not show up for larger cylinders (see Fig. 7). It was seen that these peaks are due to the interference of the terms due to $n=0,1$ and -1 that are the only significant terms for small cylinders. An experimental verification of these peaks was not possible because they occur only for very small cylinders where the received signal is low and those peaks are out of the limited 


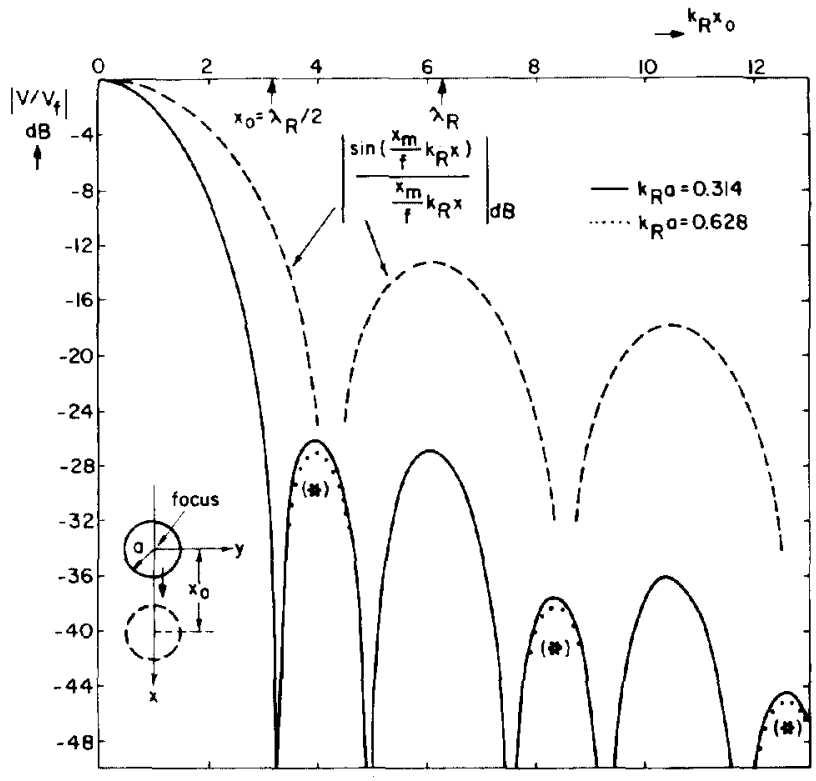

Fig. 6. Magnitude of normalized transducer output voltage as function of lateral position of cylinder. $x_{m} / f=0.74, \alpha_{T} / k_{R}=0.03, y_{0}=0.0 ; V_{f}$ is $19.84 \mathrm{~dB}$ below $V_{\text {edge }}$ for $k_{R} a=0.314$, and $9.81 \mathrm{~dB}$ below $V_{\text {edge }}$ for $k_{R} a=0.628$

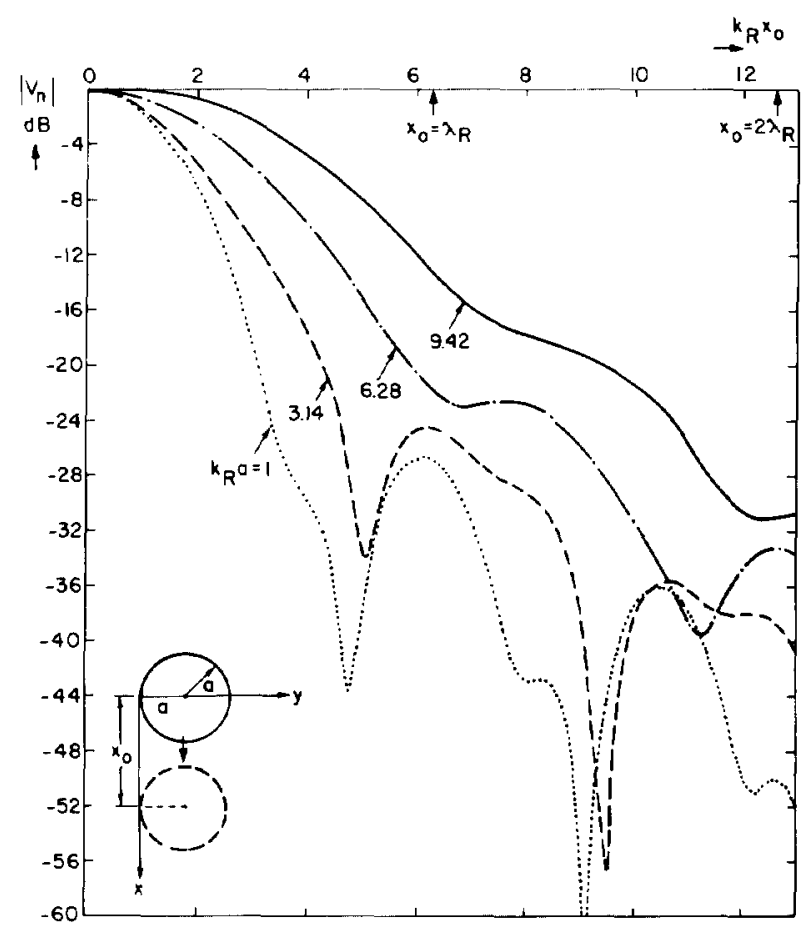

Fig. 7. Magnitude of normalized transducer output voltage as function of lateral position of cylinder, with cylinder radius as parameter. $x_{m} / f=$ $0.74, \alpha_{T} / k_{R}=0.03, y_{0}=a$; maximum voltages are below $V_{\text {edge }}$ by following amounts: $7.03 \mathrm{~dB}$ for $k_{R} a=1.0,3.01 \mathrm{~dB}$ for $k_{R} a=\pi, 1.48$ $\mathrm{dB}$ for $k_{R} a=2 \pi, 1.42 \mathrm{~dB}$ for $k_{R} a=3 \pi$.

dynamic range of the system. The other peaks in Fig. 6 correspond to the maxima of $\mid$ sinc| function as expected. It is also observed that the resolution of the system in the $x$ direction is about four times better than in the $y$ direction for the case of the aluminum sample, and this is consistent with experimental results. Note that, the resolution of the system in the $y$ direction can be improved by the use of short pulses and a time-gating arrangement [14].

The next figure, Fig. 7 , shows how the output voltage varies in the lateral direction for larger radius cylinders. Here, the cylinders are moved parallel to the $x$-axis (with their edges touching the $x$-axis), and the corresponding output voltages are plotted after being normalized by their maximum values. It is seen that for cylinders whose diameters are larger than the width of the main beam of the incident sinc function, the transducer output voltage is related rather closely to the size of the cylinder. For $k_{R} a=$ $2 \pi$ and $k_{R} a=3 \pi$, approximately $20-\mathrm{dB}$ drop in the output voltage is obtained when the cylinder is centered at the position $x_{0}=a$. Hence images obtained in such a system will correctly depict the size of the cylinders. But, for small cylinders, the response of the system is determined by the incident sinc function.

Finally, to investigate the sensitivity of the imaging system to the radius of the cylinder, the output voltages for different radii are calculated. Each cylinder is centered at the point $x_{0}=0, y_{0}=a$, where $a$ is its radius, so that the edge of each cylinder is at the focus as demonstrated in Fig. 8. The case $a \rightarrow \infty$ corresponds to an infinite straight edge reflector along $x$-axis for which the transducer output voltage is $V_{\text {edge }}$. All voltages are normalized by $V_{\text {edge }}$ and plotted versus $k_{R} a$. It is observed that the output voltage increases as the cylinder becomes larger, and for $k_{R} a=3.1\left(a \simeq \lambda_{R} / 2\right)$, the output voltage is only $3 \mathrm{~dB}$ below $V_{\text {edge }}$. This is because the incident field $u_{f}^{+}(x)$ illuminates only a finite portion along $x$-axis (when $\left|k_{R} x\right|$ $>3.5$, the value of $u_{f}^{+}(x)$ stays $13 \mathrm{~dB}$ or more below its value at $x=0$, as can be seen from Fig. 6) Thus, increasing the radius further will not change the output voltage considerably. On the other hand, for small radii the signal drops about $10 \mathrm{~dB}$ for each halving of the cylinder radius. The asymptotic line crosses the $0 \mathrm{~dB}$ axis at approximately $k_{R} a=1$.

The curve in Fig. 8 exhibits another interesting feature. As $k_{R} a$ approaches 1 from smaller values, the output voltage tends to increase faster, thus forming a local maximum at $k_{R} a=1$. This corresponds to a cylinder whose circumference is $\lambda_{R}$, i.e., the phase change around the cylinder is $2 \pi$. Such behavior can be explained by the inphase superposition of the creeping surface waves around the cylinder [35]. It is surprising that the very simple boundary conditions used in the reflection problem predicts this behavior. The dots in the Fig. 8 represent the experimental results obtained earlier [19]. Although in the experimental setup, the focal field had a wider beamwidth, the experimental curve possesses the same kind of signal enhancement around $k_{R} a=1$. For small cylinder radii the experimental and theoretical values are in good agreement. Disagreement between the curves for larger $k_{R} a$ values may be explained by the coarse approxima- 


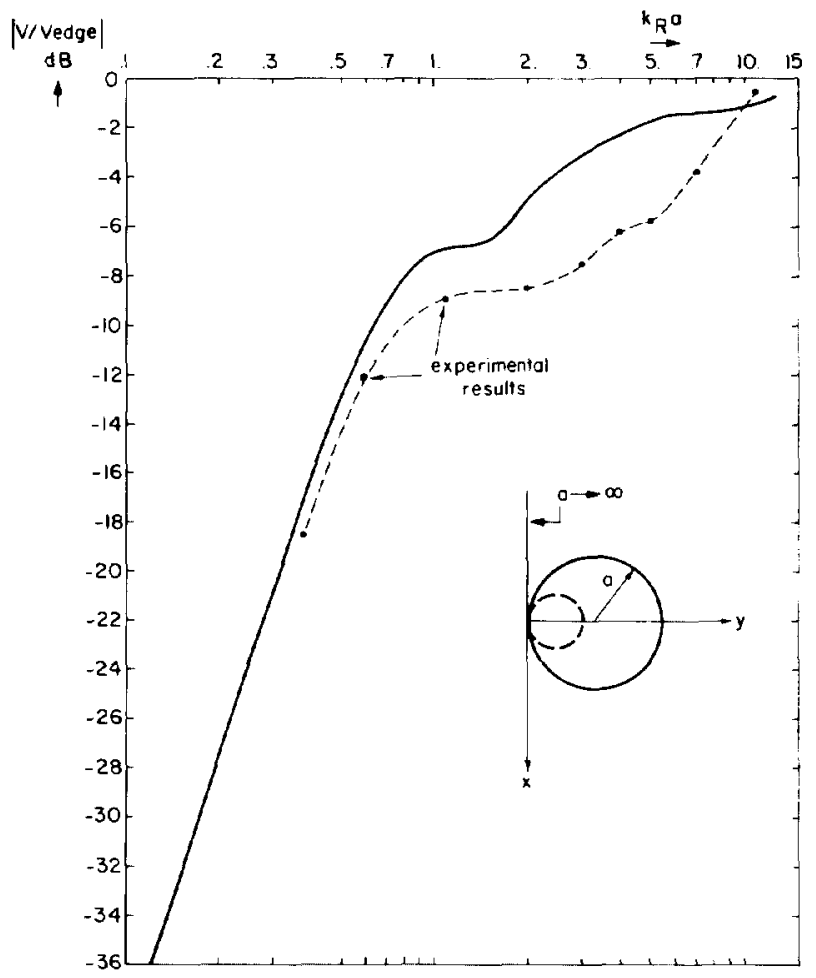

Fig. 8. Variation of normalized output voltage with cylinder radius. $x_{m} / f$ $=0.74, \alpha_{T} / k_{R}=0.03, x_{0}=0.0, y_{0}=a, V_{\text {edge }}:$ output voltage corresponding to the case $a \rightarrow \infty$.

tions made in calculation of the reflection coefficient of SAW from the cylinders, which may be radius dependent.

\section{CONCLUSION}

A theory is presented to predict the response of an imaging system making use of leaky SAW. In particular, the conical axicon SAW focusing system is considered. The response of the system to cylindrical flaws as a function of position of flaw with respect to focus point is calculated. Although the scattering of SAW from the cylindrical flaws is taken into account with a very rough approximation, the results are very consistent with experimental findings. The resolution of the system in the lateral direction is found to be very good. For a typical configuration the 3-dB width of the point response function in the lateral direction is less than a Rayleigh wavelength. But, the resolution in the axial ( $y$ ) direction is typically 3 to 4 times worse. The calculations indicate that the leak rate of SAW into the liquid medium affects the resolution of the system in the latter direction. A high-leak-rate material will result in a better resolution in the $y$ direction. It is seen that for cylindrical flaws with large radius the signal drops about $20 \mathrm{~dB}$ when the center of the cylinder is offset laterally one radius from focal point. Hence, this $20-\mathrm{dB}$ point can be used to ascertain the size of the flaws. For small size cylinders the lateral variation of the signal is the same except for the strong dependence of the signal level. Asymptomatically, there is about $10-\mathrm{dB}$ drop per halving of the flaw radius. Thus, for small cylinders the received peak amplitude can be used to determine the size of the cylinder. The presented theory also predicts an enhancement in the received signal level when the cylinder circumference equals a SAW wavelength, again consistent with experimental observations.

The response of other imaging systems utilizing leaky SAW or other leaky waves can be found with slight modification of the theory given here. Since the point response function can be calculated, standard image processing techniques such as the deconvolution operation can be utilized to improve the resolution of the images obtained with such systems.

\section{ACKNOWLEDGMENT}

The authors would like to acknowledge Dr. H. Köymen for his comments and discussions and B. Baygün and F. Sürücü for their contributions at the beginning of this study.

\section{APPENDIX}

On the Representation of Plane and Cylindrical SAW'S by Two-Dimensional Potentials

Consider an elastic medium occupying the half space for $z<0$. First assume that there is no leakage to $z>0$ region, i.e., $z=0$ is a free-surface.

A two-dimensional Rayleigh Potential $u(x, y)$ satisfying

$$
\left(\frac{\partial^{2}}{\partial x^{2}}+\frac{\partial^{2}}{\partial y^{2}}+k_{R}^{2}\right) u(x, y)=0
$$

where $k_{R}=\omega / V_{R}$ is the Rayleigh wave number can be used to represent the surface wave. The solution of (30) is in the form

$$
\exp \left(j k_{x} x\right) \exp \left(j k_{y} y\right) \text { with } k_{x}^{2}+k_{y}^{2}=k_{R}^{2} .
$$

The complete expressions for the $x, y, z$ components of the particle velocity vector, $v$, can then be written as

$$
\begin{aligned}
& v_{x}(x, y, z)=f_{1}(z) \frac{\partial}{\partial x} u(x, y) \\
& v_{y}(x, y, z)=f_{1}(z) \frac{\partial}{\partial y} u(x, y) \\
& v_{z}(x, y, z)=f_{2}(z) u(x, y) .
\end{aligned}
$$

The functions indicating the depth variation are

$$
\begin{array}{ll}
f_{1}(z)=\exp (h z)-\frac{2 h k}{k_{R}^{2}+k^{2}} \exp (k z) & \\
& z \leq 0 \\
f_{2}(z)=h \exp (h z)-\frac{2 h k_{R}^{2}}{k_{r}^{2}+k^{2}} \exp (k z) &
\end{array}
$$


where $h^{2}=k_{R}^{2}-\left(\omega / V_{L}\right)^{2}, V_{L}$ is the longitudinal wave velocity, and $k^{2}=k_{R}^{2}-\left(\omega / V_{S}\right)^{2}$ and $V_{S}$ is the shear wave velocity.

This is an exact formulation which combines shear and longitudinal waves in the solid to form the Rayleigh wave. It can be easily verified by using the particle velocity components given by (31) that

$$
T_{x z}=T_{y z}=T_{z z}=0 \text { at } z=0 .
$$

In a similar way, a two-dimensional potential satisfying

$$
\left\{\frac{\partial^{2}}{\partial \rho^{2}}+\frac{1}{\rho} \frac{\partial}{\partial \rho}+\frac{1}{\rho^{2}} \frac{\partial^{2}}{\partial \phi^{2}}+k_{R}^{2}\right\} u(\rho, \phi)=0
$$

can be used to represent cylindrical SAW's. The solution of (32) is in the form

$$
Z_{n}\left(k_{R} \rho\right) \exp (j n \phi)
$$

where $Z_{n}$ stands for the appropriate Bessel or Hankel function. The particle velocity vector is expressed as

$$
\begin{aligned}
& v_{r}=f_{1}(z) \frac{\partial}{\partial \rho} u(\rho, \phi)=f_{1}(z) k_{R} \dot{Z}_{n}\left(k_{R} \rho\right) \exp (j n \phi) \\
& v_{\phi}=f_{1}(z) \frac{1}{\rho} \frac{\partial}{\partial \phi} u(\rho, \phi)=f_{1}(z) \frac{j n}{\rho} Z_{n}\left(k_{R} \rho\right) \exp (j n \phi) \\
& v_{z}=f_{2}(z) u(\rho, \phi)=f_{2}(z) Z_{n}\left(k_{R} \rho\right) \exp (j n \phi)
\end{aligned}
$$

where $f_{1}(z)$ and $f_{2}(z)$ are same as before. It can be shown that above solutions guarantee the boundary conditions $T_{\rho z}$ $=T_{\phi z}=T_{z z}=0$ at $z=0$. Expressions equivalent to (33) can be found in literature [36].

When there is leakage to the upper half-space $(z>0)$, and dissipation in the solid, using a perturbational approach, $k_{R}$ in the $u(x, y)$ and $u(\rho, \phi)$ expressions is replaced by $k_{R}+j \alpha_{T}$; but $f_{1}(z)$ and $f_{2}(z)$ expressions can be used without any change.

\section{REFERENCES}

[1] R. J. Dewhurst, A. D. W. McKie, and S. B. Palmer, "Further evidence for two-component surface-acoustic-wave reflections from surface breaking cracks," Appl. Phys. Lett., vol. 49, pp. 1694-1695, 1986.

[2] W. Parmon and H. L. Bertoni, "Ray interpretation of the material signature in the acoustic microscope," Elect. Lett., vol. 15, pp. 685$686,1979$.

[3] K. Yamanaka and Y. Enomoto, "Observation of surface cracks with scanning acoustic microscope," J. Appl. Phys., vol. 53, pp. 846850,1982 .

[4] K. Yamanaka, Y. Enomoto, and Y. Tsuya, "Acoustic microscopy of ceramic surfaces," IEEE Trans. Son. Uttrason., vol. SU-32, pp. 313319,1985

[5] G. C. Smith and M. G. Gee, "Observation of a sub-surface defect in sapphire by Rayleigh wave reflection in the scanning-acoustic-microscope," J. Mater. Sci. Lett., vol. 5, pp. 1133-1134, 1986.

[6] A. Atalar, H. Köymen, and O. Yemişçiler, "Measurement of sensitivity of different wave modes to subsurface defects," in Proc. 1988 IEEE Ultrason. Symp., 1988.

[7] I. R. Smith, H. K. Wickramasinghe, G. W. Farnell, and C. K. Jen, "Confocal surface-acoustic-wave microscopy," Appl. Phys. Lett., vol. 42, pp. 411-413, 1983.
[8] B. Nongaillard, M. Ourak, J. M. Rouvaen, M. Houze, and E. Bridoux, "A new focusing method for nondestructive evaluation by surface-acoustic-wave," J. Appl. Phys., vol. 55. pp. 75-79, 1984.

[9] C. K. Jen, P. Cielo, J. Bussiere, F. Nadeau, and G. W. Farnell, "Phase variation of focused surface-acoustic-wave," Appl. Phys Lett., vol. 46, pp. 241-243, 1985

[10] P. Cielo, F. Nadeau, and M. Lamontagne, "Laser generation of conyergent acoustic waves for materials inspection," Ultrasonics, vol. 23, pp. 55-62, 1985.

[11] P. Cielo, C. K. Jen, and X. Maldague, "The converging surfaceacoustic-wave technique: Analysis and applications to nondestructive evaluation," Can. J. Phys., vol. 64, pp. 1324-1329, 1986.

[12] X. Maldague, P. Cielo, and C. K. Jen, "NDT applications of lasergenerated focused acoustic waves," Material Evaluation, vol. 44, pp. $1120-1124,1986$.

[13] H. Köymen and A. Atalar, "Focusing surface waves using an axicon," Appl. Phys. Lett., vol. 47, pp. 1266-1268, 1985.

[14] S. Ayter, "Focusing surface waves using conical transducers," in Proc. of 1987 IEEE Ultrason. Symp., pp. 301-304, 1987.

[15] W. P. Robbins, R. K. Mueller, and E. Rudd, "Thin-film characterization using a scanning laser acoustic microscope with surfaceacoustic-waves," IEEE Trans. Ultrason. Ferroelec. Freq. Contr. vol. 35 , pp. $477-483,1988$.

[16] A. L. Fang, "Scattering of a cylindrical wave of sound by an elastic cylinder," Acustica, vol. 13, pp. 397-402, 1963.

[17] N. N. Bojarski, "Scattering by a cylinder: A fast exact numerical solution," J. Acoust. Soc. Am., vol. 75, pp. 320-323, 1984.

[18] T. J. Cavicchi and W. D. O'Brien, "Acoustic scattering of an incident cylindrical wave by an infinite circular cylinder," IEEE Trans. Ultrason. Ferroelec. Freq. Contr., vol. 35, no 1, pp. 78-80, Jan 1988.

[19] A. Atalar and H. Köymen, "Use of a conical axicon as a surfaceacoustic-wave focusing device," IEEE Trans. Ultrason. Ferroelect. Freq. Contr., vol. UFFC 34, no. 1, pp. 53-63, Jan. 1987.

[20] J. W. Goodman, Fourier Optics. New York: McGraw-Hill, 1968.

[21] H. L. Bertoni, "Ray-Optical Evaluation of $V(Z)$ in the Reflection Acoustic Microscope," IEEE Trans. Son. Ultrason., vol. SU-31, pp. $105-116,1984$.

[22] A. Atalar and H. Köymen, " $V(Z)$ of the surface-acoustic-wave focusing system," in IEEE 1986 Ultrason. Symp. Proc., 1986, pp. 727730

[23] J. J. Stamnes, "Focusing of two-dimensional waves," J. Opt. Soc. Am., vol. 71, pp. 15-31, 1981.

[24] N. Günalp, "Transformation formulas between two-dimensional plane wave spectra and cylindrical wave expansions," to be published.

[25] F. C. Cuozzo, E. L. Cambiaggio, J.-P. Damiano, and E. Rivier, "Influence of elastic properties on Rayleigh wave scattering by normal discontinuities," IEEE Trans. Sonics. Ultrason., vol. SU-24, pp. 280-289, 1977.

[26] S. Ayter, "Scattering of Rayleigh waves from surface breaking cracks in the high frequency regime," Ph.D. Thesis, Stanford University, Stanford, CA, June 1983.

[27] Y. C. Angel and J. D. Achenbach, "Reflection and transmission of obliquely incident Rayleigh waves by a surface-breaking crack, " $J$. Acoust. Soc. Am., vol. 75, pp. 313-319, 1984.

[28] B. Q. Vu and V. K. Kinra, "Diffraction of Rayleigh waves in a halfspace. I. Normal edge crack," J. Acoust. Soc. Am., vol. 77, pp. 1425$1430,1985$.

[29] A. Gautesen, "Scattering of an obliquely incident Rayleigh wave in an elastic quarterspace," Wave Motion, vol. 8, pp. 27-41, 1986.

[30] V. K. Kinra and B. Q. Vu, "Diffraction of Rayleigh waves in a halfspace. II. Inclined edge crack," J. Acoust. Soc. Am., vol. 79, pp. $1688-1692,1986$.

[31] A. Gautesen, "Scattering of a Rayleigh wave by an elastic wedge," Wave Motion, vol. 9, pp. 51-59, 1987.

[32] P. C. Waterman, "New formulation of acoustic scattering," $J$. Acoust. Soc. Am., vol. 45, pp. 1417-1429, 1969

[33] N. Günalp (Tarhan), "Acoustic and electromagnetic scattering from cylinders buried in a half-space," Ph.D. Thesis, Middle East Tech. Univ., Ankara, Turkey, 1981.

[34] A. Atalar, "A backscattering formula for acoustic transducers," $J$. Appl. Phys., vol. 51, pp. 3093-3098, 1980.

[35] Y. H. Pao and W. Sachse, "Interpretation of time records and power spectra of scattered ultrasonic pulses in solids," J. Acoust. Soc. Am., vol. 56, pp. 1478-1486, 1974. 
[36] A. Safaai-Jazi, C.-K. Jen and G. W. Farnell, "On the excitation of time harmonic cylindrical surface-acoustic-waves." IEEE Trans. Uitrason. Ferroelec. Freq. Contr., vol. UFFC-33. no. 4. pp. 471-477. July 1986

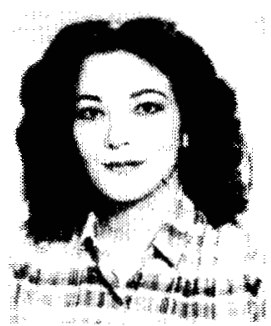

Nilgün Günalp was born in Niğde, Turkey, in 1952. She received B.S., M.S. and Ph.D. degrees in clectrical engineering from Middle East Technical University (METU), Ankara, Turkey in 1973, 1976, and 1982, respectively.

Since 1973, she has been affiliated with METU as a research assistant and instructor. From 1982 to 1984 she was with the Department of Electrical and Computer Engineering, Illinois Institute of Technology, Chicago, IL. She is now an Associate Professor at METU. Her research interests are electromagnetic and acoustic scattering, and numerical methods in electromagnetics.

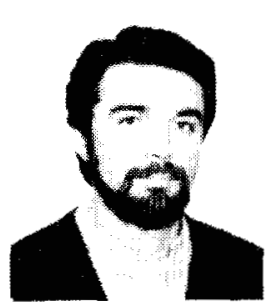

Abdullah Atalar ( $\mathrm{M}^{\circ} 88$ ) was born in Gaziantep, Turkey, in 1954. He received the B.S. degree from Middle East Technical University, Ankara. Turkey in 1974, M.S. and Ph.D. degrees from Stanford University, Stanford. CA, in 1976 and 1978 . respectively, all in electrical engineering; his thesis work was on reflection acoustic microscopy.

From 1978 to 1980 he was first a Post Doctoral

Fellow and later an Engineering Research Associate in Stanford University continuing his work on acoustic microscopy. For eight months he was with Hewlett Packard Labs, Palo Alto, CA, engaged in photoacoustics research. In 1980 he joined the Middle East Technical University as an Assistant Professor. From 1982 to 1983 on leave from University, he was with Ernst Leitz Wetzlar. Wetzlar, FRG, where he was involved in the development of the commercial acoustic microscope. He is presently an Associate Professor and Chairman of the Electrical and Electronics Engineering Department at Bilkent University, Ankara, Turkey. His current research interests include acoustic imaging, linear acoustics, and computer aided design in electrical engineering.

Dr. Atalar was awarded $\mathbf{H}$. Tuğą̧ Foundation Award of Tubitak. Turkey for his contributions to acoustic microscopy in 1984. 